\title{
PROFIL GESCHÄRFT
}

Liebe Leserin, lieber Leser,

vielleicht liegen die gesammelten Werke der ATZelektronik 2014 in diesem

Moment vor Ihnen: die Ausgaben 1 bis 6 und das Sonderheft zur Electronica, der jüngst vergangenen größten Elektronikmesse der Welt. Unser Dank gilt allen Autoren, die mit viel persönlichem Einsatz sehr wertvolle Artikel in diesem Jahr verfasst haben. Einige von Ihnen tragen in besonderem Maße zur Stärkung des Profils der ATZelektronik bei mit dem Selbstverständnis, einigen Entwicklungen in der Branche mit kritischen Blicken und etwas Abstand zu begegnen. Das ist in diesem Jahr Dank mutiger und engagierter Gesprächspartner zudem in Interviews gut gelungen.

Gemeinsam ließen wir uns nicht von Hypes leiten. Beispielsweise haben wir Elektromobilitätsprojekte frühzeitig hinterfragt und uns mit Bodenhaftung auf die Hausaufgaben im Bordnetz und Antriebsstrang konzentriert. Mit den Ergebnissen gibt es zum Beispiel nun erstmals fundierte Berechnungen, wann und wie die Kosten von Akkumulatoren sinken können und dass deutsche Firmen dabei eine untergeordnete Rolle spielen, wie in dem Report Seite 30 resümiert.

Eine neue Welle zeichnet sich ab: das vernetzte Fahrzeug mit dem Ziel des autonom fahrenden Automobils. Hier hinterfragen wir die Zeitpläne der allzu optimistischen Roadmap in Richtung 2025, ohne die Entwicklungen an sich infrage zu stellen. Dies tun wir redaktionell und zusätzlich im Rahmen der 1. Internationalen ATZ-Fachtagung Fahrerassistenz im April 2015 in Frankfurt am Main. Ein Report in ATZelektronik 5 fasste bereits die entscheidenden Erkenntnisse zusammen, die im Jahr 2014 mithilfe von Experten gereift sind.

Fahrerassistenzsysteme rücken auch immer stärker in den Fokus der Integration von Consumer-Elektronik ins Auto- mobil. Diese birgt Gefahren, auf die der ZVEI und die Halbleiterhersteller in einer Kommunikationsoffensive 2014 hinweisen. Mit vier aufeinander aufbauenden Autorenbeiträgen in mehreren Ausgaben der ATZelektronik wurde das Thema in diesem Jahr beleuchtet. Zwei Interviews objektivieren die Sachverhalte, die die Industrien beachten müssen - technisch und organisatorisch, unter anderem in neuen Zusammenarbeitsformen. Das zweite Interview lesen Sie auf Seite 20 in dieser Ausgabe, in dem mit Audi erstmals ein OEM öffentlich Stellung bezieht.

Zum Profil der ATZelektronik zählt in erster Linie die technische und ausführliche fachliche Tiefe von Artikeln. Da, wo es angebracht ist, gilt es aber auch die Arbeit der Entwickler unter Marketingaspekten und Kostenrechnungen zu beleuchten, um Fehleinschätzungen zu vermeiden und Enttäuschungen vorzubeugen. So soll uns dieses Editorial die Perspektive für unsere Zusammenarbeit in 2015 geben.

Ich freue mich auf ein gutes neues Jahr mit Ihnen

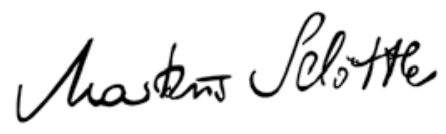

\section{MARKUS SCHÖTTLE,}

Stellvertretender Chefredakteur Wiesbaden, 9. November 2014

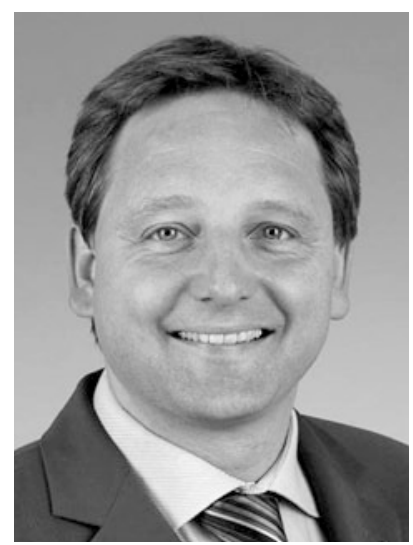

